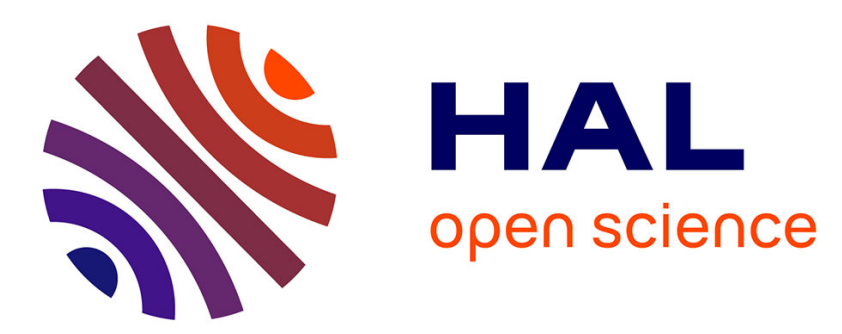

\title{
Structure of the water/surfactant interface in micelles : an NMR study of SDS micelles labelled with paramagnetic ions \\ B. Cabane
}

\section{> To cite this version:}

B. Cabane. Structure of the water/surfactant interface in micelles: an NMR study of SDS micelles labelled with paramagnetic ions. Journal de Physique, 1981, 42 (6), pp.847-859. 10.1051/jphys:01981004206084700 . jpa-00209070

\section{HAL Id: jpa-00209070 https://hal.science/jpa-00209070}

Submitted on 1 Jan 1981

HAL is a multi-disciplinary open access archive for the deposit and dissemination of scientific research documents, whether they are published or not. The documents may come from teaching and research institutions in France or abroad, or from public or private research centers.
L'archive ouverte pluridisciplinaire HAL, est destinée au dépôt et à la diffusion de documents scientifiques de niveau recherche, publiés ou non, émanant des établissements d'enseignement et de recherche français ou étrangers, des laboratoires publics ou privés. 


\title{
Structure of the water/surfactant interface in micelles : an NMR study of SDS micelles labelled with paramagnetic ions
}

\author{
B. Cabane \\ Laboratoire de Physique des Solides $\left({ }^{*}\right)$, Université Paris-Sud, 91405 Orsay, France
}

(Reçu le $1^{\mathrm{er}}$ septembre 1980, accepté le 19 février 1981)

\begin{abstract}
Résumé. - On présente une méthode nouvelle pour déterminer la structure de l'interface eau/surfactant et la configuration des molécules dans les micelles de surfactant. Cette méthode consiste à observer par RMN les interactions dipolaires entre les noyaux des molécules de surfactant et des contreions paramagnétiques adsorbés sur la surface de ces micelles. L'expérience compare les vitesses de relaxation de noyaux appartenant à différents groupes chimiques dans les molécules de surfactant ; dans certains cas simples ces vitesses de relaxation dépendent seulement des distances entre les noyaux et les ions. Ainsi les vitesses de relaxation mesurées peuvent être reliées aux populations des noyaux dans des couches situées à diverses profondeurs de la surface. Les résultats mènent à la discuscussion suivante pour la structure de ces couches successives : (i) couches de surface contenant les charges hydratées (sont-elles lisses ou rugueuses ?); (ii) couches de contact eau/hydrocarbone (jusqu'à quelle profondeur l'eau pénètre-t-elle dans la micelle ?); (iii) couches du cour hydrocarboné (quel est le degré d'organisation des chaînes du surfactant ?).
\end{abstract}

\begin{abstract}
A method has been developed for determining the structure of the water/surfactant interface and the configuration of the surfactant molecules in micelles. In this method, NMR is used to measure the magnetic dipolar interactions between the nuclei of the surfactant molecules and paramagnetic counterions adsorbed on the surface of the micelles. The experiment compares the relaxation rates of nuclei belonging to different chemical groups in the surfactant molecules; in some simple cases these relaxation rates only depend on the distances between the nuclei and the ions. Then the measured relaxation rates can be related to the populations of the nuclei in layers located at various depths from the paramagnetic surface. According to the data, the structure of these successive layers in a spherical micelle can be discussed in the following terms :(i) outermost layers containing the hydrated charges (how smooth or regular are they ?); (ii) layers of water/hydrocarbon contact (how deep does water penetrate in the micelle ?); (iii) layers of the hydrocarbon core (how disorganized is the packing of the surfactant chains ?).
\end{abstract}

Introduction. - There has been considerable debate about the structure of micelles. Nevertheless, forty years after the work of Hartley [1], who first recognized that they were small spherical aggregates of surfactant molecules, many features of their structure are still unknown to us. For example, we do not know whether the charged surface of a micelle is smooth or irregular (do some charged groups protrude above or stay below the surface ?). Nor do we know the thickness of the water/hydrocarbon interface (is water-hydrocarbon contact limited to a thin layer, or is there substantial water penetration between the aliphatic chains of the surfactant molecules ?). Neither is there much agreement about the configuration of these aliphatic chains (thermodynamic evidence suggests that they resemble liquid paraffins,

(*) Laboratoire associé au C.N.R.S. but most textbook pictures of micelles show rigid radial chains).

So far, the main body of structural information has been provided by scattering techniques. These have provided a determination of the size, shape, and charge of the micelles [2-7]. At larger angles, the diffuse band corresponding to the average distance between two surfactant chains has been observed by Luzzati et al. [6]. They concluded that in micelles these chains are in a conformational state similar to that of liquid paraffins. Unfortunately, due to an insufficient resolution $(10$ to $20 \AA)$, the information about the water/ surfactant interface obtained through these techniques has been limited to an overall thickness of the whole polar region of the micelles.

The spectroscopy of the surfactant molecules (NMR [8-10], Raman [11, 12]) can provide a high resolution (individual $\mathrm{CH}_{2}$ groups or individual vibration modes), but the results use the molecule and not 
the micelle as a geometrical frame of reference. For example, one may find that the first $4 \mathrm{CH}_{2}$ groups of the surfactant molecules are hydrated, and conclude that water penetrates the micellar core over a depth of $5 \AA$. This conclusion would only be correct if the surfactant chains would extend radially from the centre to the surface of the micelle. There is considerable evidence that they do not; if an orientational order parameter is defined for these chains by analogy with the anisotropic phases [13], its typical value in micelles is about 0.2 . This implies that the chains of the surfactant molecules in micelles have few radial segments; consequently we do not know a priori what is the average depth in the micelle of a given group (say, the 4th $\mathrm{CH}_{2}$ of each chain). Thus the disorder of the molecules prevents us from taking information which is related to the molecular frame of reference and expressing it into the micellar frame of reference.

The technique described here consists in labelling the surface of a micelle with paramagnetic ions, and observing through NMR the effect of these ions on the various $\mathrm{CH}_{2}$ groups of the surfactant molecules. (For a general discussion of the use of paramagnetic ions in NMR, see $[14,15]$.) Therefore it combines the high resolution of spectroscopic techniques with the use of the charged surface of the micelle as a geometrical frame of reference. A few results obtained through this technique have been published already [16-19]. This paper presents a somewhat more elaborated discussion, and a full set of data for dodecylsulfate surfactants with sodium (SDS), cobalt, and manganese as counterions; it is organized in the following way :

- Section 1 describes the experimental procedures (labelling of SDS micelles with $\mathrm{Co}^{++}$and $\mathrm{Mn}^{++}$ ions, magnetic resonance of the dodecylsulfate ions).

- Section 2 describes what the " surface labelling " technique measures (all the possible interactions between the observed nuclei and the paramagnetic ions are discussed).

- Section 3 presents the experimental data. Most of these results are used only to show that in $\operatorname{Co}(\mathrm{DS})_{2}$ and $\mathrm{Mn}(\mathrm{DS})_{2}$ micelles the situation is particularly simple. In such cases, all the data can be expressed as values of a single geometrical parameter, and represented by a single curve.

- Section 4 discusses the consequences of these results for the geometry of the water/surfactant interface in micelles, and presents an « onion shell » model for the packing of surfactant chains in micelles.

1. Experimental procedures and physico-chemical background. - 1.1 MATERIALs. - The micellar solutions were made with sodium dodecylsulfate (SDS) and various amounts of cobalt and manganese chlorides in $\mathrm{D}_{2} \mathrm{O}$. SDS was $\mathrm{BDH}$ « specially purified for biochemistry » grade; $\mathrm{CoCl}_{2}$ and $\mathrm{MnCl}_{2}$ were Prolabo «Normapur" reagents; $\mathrm{D}_{2} \mathrm{O}$ was bought from CEA, France (isotopic purity $99.8 \%$ ).
It has been shown that in a micellar solution containing monovalent $\left(\mathrm{Na}^{+}\right)$and divalent $\left(\mathrm{Mn}^{++}\right)$counterions the divalent ions are preferentially adsorbed on the micellar surface $[20,21]$. For example, the critical micelle concentration (c.m.c.) of surfactant mixtures SDS $+\mathrm{Mn}(\mathrm{DS})_{2}$ decreases rapidly from

$$
8.25 \times 10^{-3} \text { to } 2.7 \times 10^{-3} \mathrm{~mole} / \text { litre }
$$

when the mole fraction of $\mathrm{Mn}(\mathrm{DS})_{2}$ increases from 0 to 0.2 ; it subsequently decreases much slower to $1.2 \times 10^{-3}$ for pure $\mathrm{Mn}(\mathrm{DS})_{2}$.

Light scattering provides a determination of the charge and of the aggregation number of the micelles. For $\mathrm{Co}(\mathrm{DS})_{2}$, the fraction of $\mathrm{Co}^{++}$ions which are not condensed on the micelles is equal to 0.11 ; the molecular weight of these micelles is 27400 , corresponding to an aggregation number of 103 ; adding $0.1 \mathrm{~mole} /$ litre of $\mathrm{CoSO}_{4}$ only increases the molecular weight to 30300 [20]. It can therefore be concluded that solutions containing $10^{-2}$ to $10^{-1}$ mole/litre of SDS with various amounts of $\mathrm{Co}^{++}$or $\mathrm{Mn}^{++}$ions form micelles with an aggregation number which is close to 100 ; this is comparable to the maximum aggregation number for globular SDS micelles [22, 23]. Finally, these micelles are also in equilibrium with a small number of unassociated $\mathrm{DS}^{-}$ions; the concentration of these unassociated ions is close to the c.m.c., and therefore much smaller than the concentrations of micellized material for the solutions studied here.

1.2 Magnetic Resonance OF THE $\mathrm{DS}^{-}$IONS. The NMR experiments were performed on the $270 \mathrm{MHz}$ FT instrument of the Groupe de Biophysique, Ecole Polytechnique, 91120 Palaiseau, France. With this instrument all the ${ }^{13} \mathrm{C}$ lines of the SDS spectrum could be resolved, except for 3 carbons in the middle of the chain. In order to lift the ambiguity concerning the assignment of these lines [19, $24,25]$, a sample was synthesized which was selectively deuterated on the $3^{d}$ carbon after the head group [26]. The intensity and shape of the deuterated carbon's line are drastically modified; thus that line was easily assigned ; then the lines of the $4^{\text {th }}$ and $5^{\text {th }}$ carbons were assigned through their isotopic shifts. The other lines were assigned through their positions and relaxation times [24]. The resulting assignment is shown in table I. All the ${ }^{13} \mathrm{C}$ measurements were made with ${ }^{1} \mathbf{H}$ decoupling; unless stated otherwise, the temperature was kept constant at $22 \pm 1 \mathrm{C}$. $T_{1}$ measurements were performed with a saturation « comb » followed, after a time interval, by a measurement pulse. Line broadenings caused by the paramagnetic ions were measured by fitting the observed line shape to a line of the same carbon in a reference spectrum which had been broadened by the computer.

2. Nuclear magnetic relaxation by paramagnetic ions adsorbed on micelles. - The nuclei of the surfactant molecules interact with the magnetic moments 
of the adsorbed paramagnetic ions. This interaction depends upon the mode of adsorption of the ions on the micellar surface. One can distinguish between adsorption with and without dehydration of the ions.

2.1 AdSORPTION Without DEHYDRATION. - In this case the paramagnetic ions and the polar groups of the surfactant molecules remain separated by the water molecules of their hydration shells. Thus the ions are only weakly adsorbed, and the correlation time for their adsorption-desorption processes is short [27]. Consequently the nuclei will only be sensitive to the average density of paramagnetic ions at the surface of the micelle, and not to the instantaneous position of an ion. Moreover, the local environment of an adsorbed paramagnetic ion remains isotropic; therefore its magnetic susceptibility tensor is isotropic as well; for this reason no dipolar "pseudocontact " shifts are observed. Finally, and most important, the wavefunction of the paramagnetic electron remains localized on the ion and its solvation shell ; it does not extend over the surfactant molecules. This prevents a « contact " interaction between the electron and the observed nuclei.

In summary, the nuclei will experience purely dipolar interactions produced by a density of paramagnetic ions on the micellar surface; as these interactions have no static components, they only affect the nuclei through their fluctuations in time. They are modulated by 3 types of motions : rotations of the ion-nucleus axis, variations of the ion-nucleus distance, and fluctuations of the spin of the paramagnetic electron. Things are simple when the fluctuations of the electronic spin are faster than the other motions, and therefore control the fluctuations of the dipolar interactions. In this case, the dipolar interactions contribute to the relaxation rates of the observed nuclei in a simple way, as shown by the relevant version of the Solomon-Bloembergen equations $[28,29]$ :

$$
\begin{aligned}
& \frac{1}{T_{1}}=\frac{D}{d^{6}}\left(\frac{6 \tau_{\mathrm{s}}}{1+\omega_{\mathrm{I}}^{2} \tau_{\mathrm{s}}^{2}}+\frac{14 \tau_{\mathrm{s}}}{1+\omega_{\mathrm{s}}^{2} \tau_{\mathrm{s}}^{2}}\right) \\
& \frac{1}{T_{2}}=\frac{D}{d^{6}}\left(4 \tau_{\mathrm{s}}+\frac{3 \tau_{\mathrm{s}}}{1+\omega_{\mathrm{l}}^{2} \tau_{\mathrm{s}}^{2}}+\frac{13 \tau_{\mathrm{s}}}{1+\omega_{\mathrm{s}}^{2} \tau_{\mathrm{s}}^{2}}\right)
\end{aligned}
$$

where $D$ is a constant related to the magnetic moments, $\omega_{\mathrm{I}}$ is the Larmor frequency of the observed nucleus, $\omega_{\mathrm{s}}$ the Larmor frequency of the paramagnetic electron, and $\tau_{\mathrm{s}}$ its spin relaxation time.

The geometrical information provided by these relaxation rates is contained in their $1 / d^{6}$ dependence on the ion-nucleus distance. As the motions of the ion and nucleus are quite fast compared to NMR time scales [27], the relaxation rates measure an average over all their possible locations in the micelle (Fig. 1). In particular, it does not matter whether or not the observed nucleus belongs to a molecule which is bound to the ion, as dipolar interactions are trans- mitted through space and not through the bonds of the molecule. Thus a measure of the average distance $\left\langle 1 / d^{6}\right\rangle$ between the nucleus and the paramagnetic surface is obtained; this is the structural information which is used in this paper.

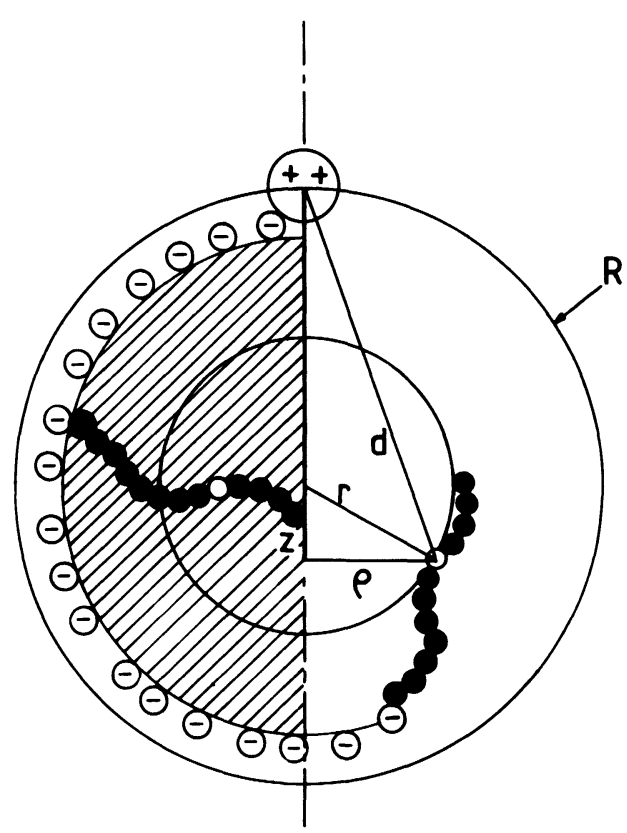

Fig. 1. - Geometry for the dipolar interaction between a nucleus in a micelle and a paramagnetic ion on the surface of this micelle. In the case of ions with fast electron spin relaxation times, the magnetic relaxation rate of the nucleus measures an average of this interaction over all possible locations of the ion and nucleus in the micelle, i.e. an average of $1 / d^{6}$.

2.2 AdSORPTION WITH DEHYDRATION. - In this case the paramagnetic ion loses some of the water molecules of its solvation shell and binds directly to the polar group of surfactant molecule. For this reason the wavefunction of the paramagnetic electron extends over parts of the surfactant molecule; there is a contact interaction between this electron and some nuclei of the surfactant, producing large contributions to the shifts and relaxation rates of these nuclei. As this interaction is transmitted through chemical bonds, only molecules bound to paramagnetic ions are affected. If the adsorption-desorption processes of the ions were slow compared to NMR time scales, one could reach the slow exchange limit where the nuclei of surfactant molecules bound to paramagnetic ions give NMR lines separate from those which belong to the other molecules [30]. However, for all the surfactants studied so far (sulfates, carboxylates), the adsorption times are much too short for this, and one is in the exchange regime where the NMR lines correspond to a time average over surfactant molecules bound directly and unbound to paramagnetic ions. Finally, it is worth mentioning that in this case of strong adsorption the susceptibility tensor of the ion is anisotropic; therefore the dipolar interaction between the nuclei and the ion has some static components which are not averaged out by 
rotational motions; for this reason, dipolar « pseudocontact » shifts can be observed in addition to the contact shifts mentioned above.

In summary, this type of adsorption produces large shifts, both contact and dipolar in origin, on the observed nuclei; there is also a large "contact » contribution to the relaxation rates, as shown by the Solomon-Bloembergen equations for this case $[28,29]$ :

$$
\begin{aligned}
& \frac{1}{T_{1}}=\frac{D}{d^{6}}\left(\frac{6 \tau_{\mathrm{s}}}{1+\omega_{\mathrm{l}}^{2} \tau_{\mathrm{s}}^{2}}+\frac{14 \tau_{\mathrm{s}}}{1+\omega_{\mathrm{s}}^{2} \tau_{\mathrm{s}}^{2}}\right)+C\left(\frac{\tau_{\mathrm{s}}}{1+\omega_{\mathrm{s}}^{2} \tau_{\mathrm{s}}^{2}}\right) \\
& \frac{1}{T_{2}}=\frac{D}{d^{6}}\left(4 \tau_{\mathrm{s}}+\frac{3 \tau_{\mathrm{s}}}{1+\omega_{\mathrm{I}}^{2} \tau_{\mathrm{s}}^{2}}+\frac{13 \tau_{\mathrm{s}}}{1+\omega_{\mathrm{s}}^{2} \tau_{\mathrm{s}}^{2}}\right)+ \\
& +\frac{C}{2}\left(\tau_{\mathrm{s}}+\frac{\tau_{\mathrm{s}}}{1+\omega_{\mathrm{s}}^{2} \tau_{\mathrm{s}}^{2}}\right)
\end{aligned}
$$

where $C$ is related to the hyperfine constant for the contact interaction and as above it is assumed that the fluctuations of the electronic spin control the correlation times of the dipolar and contact interactions. For ions such as $\mathrm{Mn}^{++}$, which have a long $\tau_{\mathrm{s}}$, the contact term contributes much more to $1 / T_{2}$ than to $1 / T_{1}$; thus large values of $T_{1} / T_{2}$ are a good indicator for the presence of contact interactions [34]. For $\mathrm{Co}^{++}$, another effect may occur : if the paramagnetic shifts are large enough, the chemical exchange of the ions between adsorption sites can dominate the linewidths (see App. A). It is therefore quite difficult to extract geometrical information from the values of $1 / T_{2}$ in the strong adsorption case. It often happens, however, that the values of $1 / T_{1}$ are still dominated by the dipolar term, in which case one can still extract the average value of $1 / d^{6}$.

2.3 Artefacts. - 2.3.1 Spin diffusion. - So far we have seen 2 routes (dipolar and contact) for the interaction between a nucleus and a paramagnetic ion. In anisotropic or viscous systems there is another possible route : this is by a succession of mutual spin flops along the molecule, from the site of adsorption of the ion to the observed nucleus [14]. In anisotropic systems (e.g. liquid crystals) this process leads to a uniform spin temperature for all the ${ }^{1} \mathrm{H}$ nuclei ; consequently, the relaxation rate of a given nucleus no longer measures its spatial distance to the paramagnetic ion. In isotropic solutions this effect is reduced by a factor equal to the ratio of the nuclear spin-spin interaction frequency to the frequency of the rotational motions. A good estimate for the magnitude of this effect can be obtained by observing whether, in the absence of paramagnetic ions, all the ${ }^{1} \mathrm{H}$ nuclei have the same relaxation rate. For the case of ${ }^{13} \mathrm{C} \mathrm{NMR}$, the proton decoupling irradiation cancels the magnetization of the protons, and prevents the spin diffusion between protons from affecting the ${ }^{13} \mathrm{C}$ relaxation rates. Thus the ${ }^{13} \mathrm{C}$ relaxation rates are free from this artefact.
2.3.2 Overall deformation of the micelle. - For many surfactants, there is a range of concentrations and ionic strengths for which the micelles are globular and tend to retain a constant shape and size [22, 23]. However there are other conditions (very close to the c.m.c., or in the vicinity of the sphere cylinder transition) where the size and shape of the micelles are very sensitive to external conditions. In these cases the addition of even one multivalent ion per micelle can have drastic consequences : for example, in a concentrated micellar solution it may displace the sphere-cylinder equilibrium of the micelles. Consequently, the rotation times of these micelles will be slower; this will produce a large effect on the transverse relaxation rates of the nuclei, and this effect is unrelated to the magnetic ion - nucleus interactions. It is therefore desirable to work in a range of concentrations and ionic strengths where the micelle shape is stable ; in doubt, the effect of the paramagnetic ions can be compared with that of non magnetic multivalent ions.

\subsubsection{Local deformation of the micellar surface. -} Some micelles have a surface whose curvature is not uniform (spherocylinders...). It may be that, at low concentrations in paramagnetic ions, these ions will be adsorbed at special sites on the micelle, where the curvature differs from the average curvature of the surface. Or the paramagnetic ions themselves may produce a local deformation of the micellar surface. In both cases, the geometry which will be observed around one ion is atypical; it does not represent the average geometry of the micellar surface. There is a simple remedy : if possible, study micelles for which all the counterions are paramagnetic; then compare the results with those for micelles with only one or two paramagnetic counterions; this has been done in the present work.

2.3.4 Effect of relative motions of the ion and nucleus. - So far it has been assumed that the fluctuations of the electronic spin are fast enough to control the fluctuations of the dipolar electronnucleus interaction. This is certainly the case for ions with short electronic relaxation times, such as $\mathrm{Co}^{++}\left(\tau_{\mathrm{e}}=10^{-12}\right.$ to $\left.10^{-13} \mathrm{~s}\right)$ [31-33]. On the other hand, it may not be the case for $\mathrm{Mn}^{++}\left(\tau_{\mathrm{e}}=10^{-8}\right.$ to $\left.10^{-9} \mathrm{~s}\right)$ or $\mathrm{Gd}^{+++}\left(\tau_{\mathrm{e}}=10^{-7}\right.$ to $\left.10^{-8} \mathrm{~s}\right)$ [31-33]. If the fluctuations of the electronic spin are too slow, then the fluctuations of the dipolar ion-nucleus interactions will be controlled by the relative motions of the ion and the nucleus. In this case the simplified equations given above for the nuclear relaxation rates no longer apply; instead one has to calculate the exact spectral densities for the dipolar interactions, which are Fourier transforms of the correlation functions :

$$
G_{m}(t)=\left\langle\frac{Y_{2 m}(r(0)) Y_{2 m}(r(t))}{r^{3}(0) r^{3}(t)}\right\rangle .
$$


This problem has been treated in the case of a planar geometry by Bloom, McKay and Abdolall [35]. In general it is found that the relaxation rates still depend upon the average distance between the ion and the nucleus, but this dependence is not as strong as in the case where the fluctuations of the electronic spin are dominant. For this reason it is very important to establish which time controls the fluctuations of the dipolar interactions. A good way to do it is to compare the relaxation rates obtained with $\mathrm{Co}^{++}$, $\mathrm{Mn}^{++}$and $\mathrm{Gd}^{+++}$ions. If these relaxation rates simply scale up with the electronic spin relaxation time $\tau_{e}$, then it is safe to assume that the fluctuations of the electronic spin are dominant. On the other hand, if different patterns of relaxation rates are obtained with different ions (for example if the ratio of the relaxation rates of 2 types of nuclei changes when the ion is changed) the simple analysis given in 2.1 and 2.2 does not apply.

3. Experimental data. - 3.1 TyPICAL RESUlT. Figure 2 presents the values of the relaxation rates produced by $\mathrm{Co}^{++}$ions on the ${ }^{13} \mathrm{C}$ nuclei of SDS molecules. It will be argued later that the source of these relaxation rates is the modulation of the dipolar ion-nucleus interactions by the fluctuations of the electronic spin. For this reason they measure the value of $1 / d^{6}$, averaged over all positions and configurations of a SDS molecule in a micelle; this yields an average depth for the observed nucleus in the micellar core. The data show an initial drop by a factor of 3 , followed by a «plateau ". This is somewhat surprising, as it was expected that the values of $\left\langle 1 / d^{6}\right\rangle$ would decrease by more than one order of magnitude from the head to the tail of the chain. Thus it is necessary to find out whether this behaviour is general, and look for possible explanations.

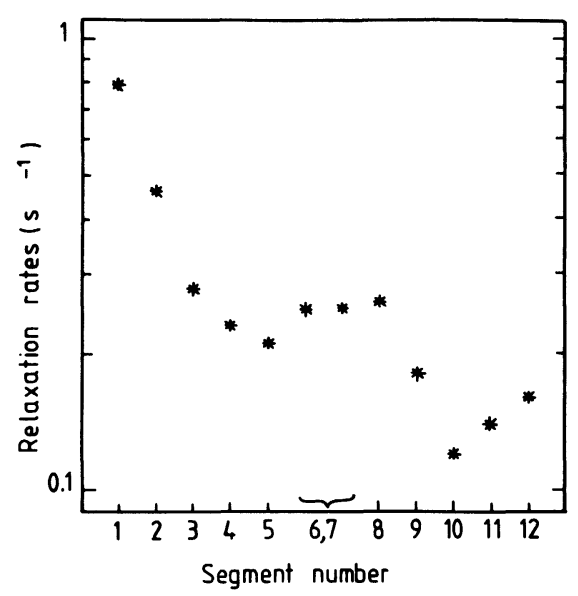

Fig. 2. - Relaxation rates produced by $\mathrm{Co}^{++}$ions on the ${ }^{13} \mathrm{C}$ of the SDS molecules in a 0.165 molar solution. The data plotted here correspond to the difference between the values of the relaxation rates in SDS micelles covered with paramagnetic $\mathrm{Co}^{++}$ions and those in SDS micelles covered with diamagnetic $\mathrm{Mg}^{++}$ions.

3.2 ${ }^{1} \mathrm{H}$ RELAXATION RATES. - The first parameter whose effect was investigated is the composition in paramagnetic ions per micelle. This required measurements on micelles saturated with paramagnetic ions as well as micelles with only one or two paramagnetic ions. $\mathrm{Co}^{++}$ions were used, as they have very short electronic relaxation times; thus even large numbers of them only produce moderate broadening of the NMR lines. Figure 3 shows the raw data, i.e. the growth of the total longitudinal relaxation rate of each ${ }^{1} \mathrm{H}$ line upon addition of $\mathrm{Co}^{++}$. The useful experimental quantities are the ratios of the " paramagnetic » contributions to these relaxation rates (i.e. contribution of the $\mathrm{Co}^{++}$ions to the relaxation rate of $\mathrm{C} 3 /$ contribution of the $\mathrm{Co}^{++}$ions to the relaxation rate of $\mathrm{C1}$ ). Figure 4 shows that these ratios do not depend upon the composition in $\mathrm{Co}^{++} /$micelle.

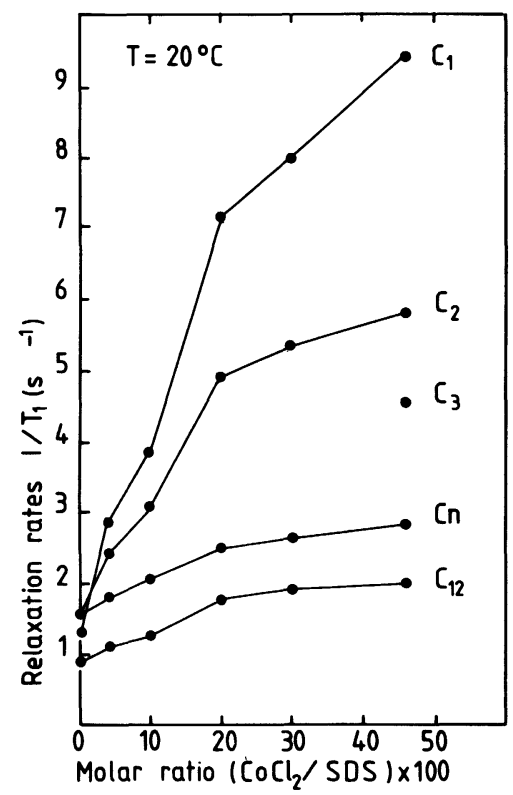

Fig 3. - Effect of added $\mathrm{Co}^{++}$ions on $1 / T_{1}$ for ${ }^{1} \mathrm{H}$ nuclei in SDS micelles $\left(0.034\right.$ molar SDS solution at $\left.20^{\circ} \mathrm{C}\right)$. The curvature is caused by the progressive saturation of the micellar surface with $\mathrm{Co}^{++}$ions.

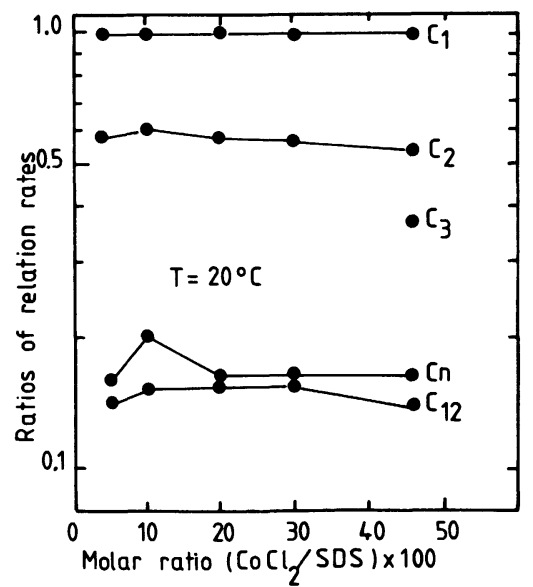

Fig. 4. - Ratios of the contributions of $\mathrm{Co}^{++}$ions to $1 / T_{1}$ for ${ }^{1} \mathrm{H}$ nuclei in SDS micelles (i.e. contribution of $\mathrm{Co}^{++}$to $1 / T_{1}$ for $\mathrm{C} 2$ divided by their contribution to $1 / T_{1}$ for $\mathrm{C} 1$ ). Notice that these ratios are the same for micelles with few $\mathrm{Co}^{++}$ions and for micelles saturated with $\mathrm{Co}^{++}$. 
Increasing the temperature from 20 to $60^{\circ} \mathrm{C}$ only produces a very slight decrease in all the relaxation rates (Figs. 5 and 6). At this point we can conclude that the relaxation rates are small compared with those for known complexes of $\mathrm{Co}^{++}$ions [31-33], that the effect of the composition in $\mathrm{Co}^{++}$ions per micelle is trivial, and that the effect of temperature is very weak.

The other set of ${ }^{1} \mathrm{H}$ data concerns SDS micelles with adsorbed $\mathrm{Mn}^{++}$ions. As these ions have long electronic spin relaxation times, they produce faster relaxation rates of the nuclei. Figure 7 shows that the measured rates are about 220 times larger than with $\mathrm{Co}^{++}$ions (they are also small compared with those for known complexes of $\mathrm{Mn}^{++}$ions [31-33]). The ratios of these relaxation rates for different $\mathrm{CH}_{2}$ groups of the SDS molecule are also independent from the composition in $\mathrm{Mn}^{++}$ions per micelle; they are identical with the ratios measured with $\mathrm{Co}^{++}$ions (Fig. 8). Thus the effect of changing the paramagnetic ion is to scale up all the relaxation rates in the same way. Finally the ratio of transverse

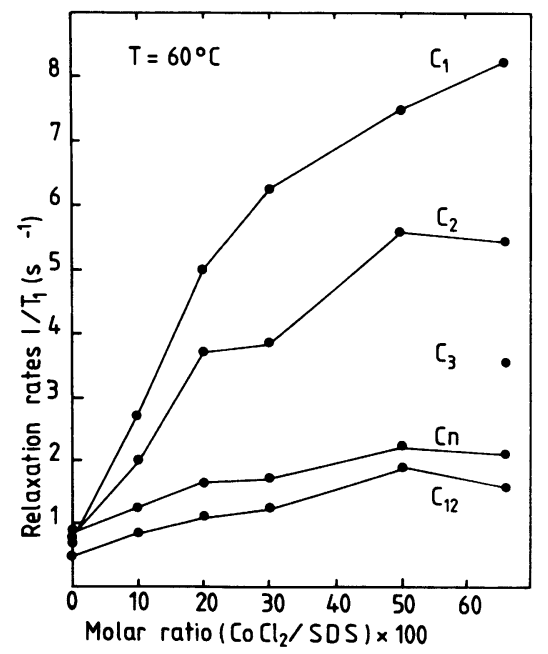

Fig. 5. - Effect of added $\mathrm{Co}^{++}$ions on $1 / T_{1}$ for ${ }^{1} \mathrm{H}$ nuclei in SDS micelles at $60^{\circ} \mathrm{C}$ (compare with Fig. 3).

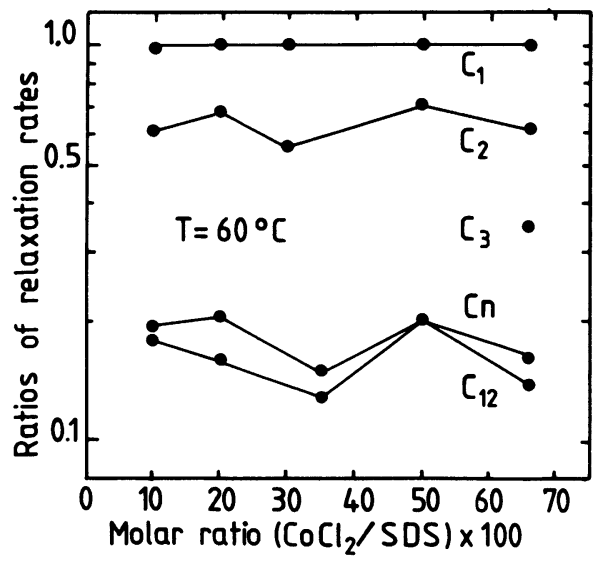

Fig. 6. - Ratios of the contributions of $\mathrm{Co}^{++}$ions to $1 / T_{1}$ for ${ }^{1} \mathrm{H}$ nuclei in SDS micelles at $60^{\circ} \mathrm{C}$ (compare with Fig. 4).

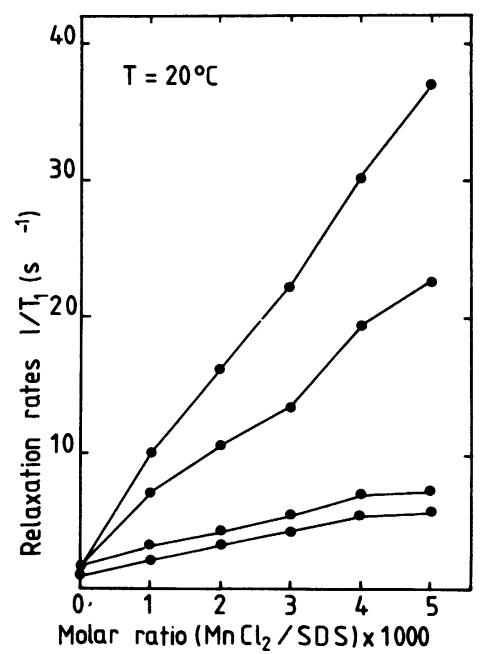

Fig. 7. - Effect of $\mathrm{Mn}^{++}$ions on $1 / T_{1}$ for ${ }^{1} \mathrm{H}$ nuclei in SDS micelles $\left(0.034\right.$ molar SDS solution at $\left.20^{\circ} \mathrm{C}\right)$. The effect per $\mathrm{Mn}^{++}$ion is 220 times larger than with $\mathrm{Co}^{++}$(Fig. 3).

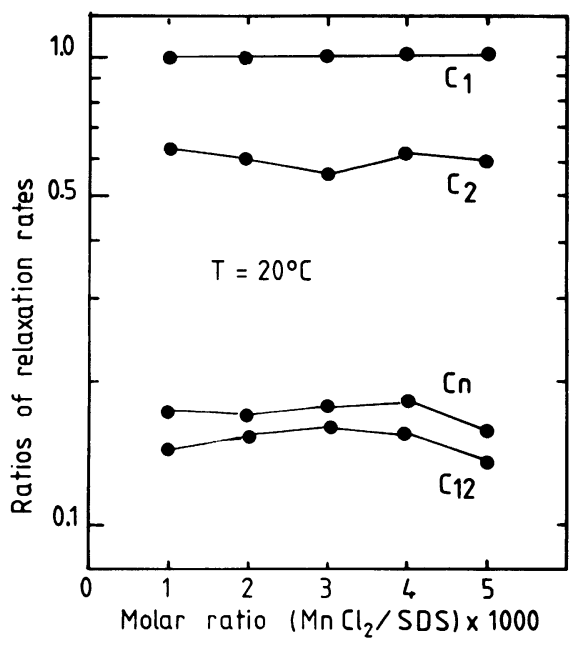

Fig. 8. - Ratios of the contributions of $\mathrm{Mn}^{++}$to $1 / T_{1}$ for ${ }^{1} \mathrm{H}$ nuclei in SDS micelles at $20^{\circ} \mathrm{C}$ (compare with Fig. 4).

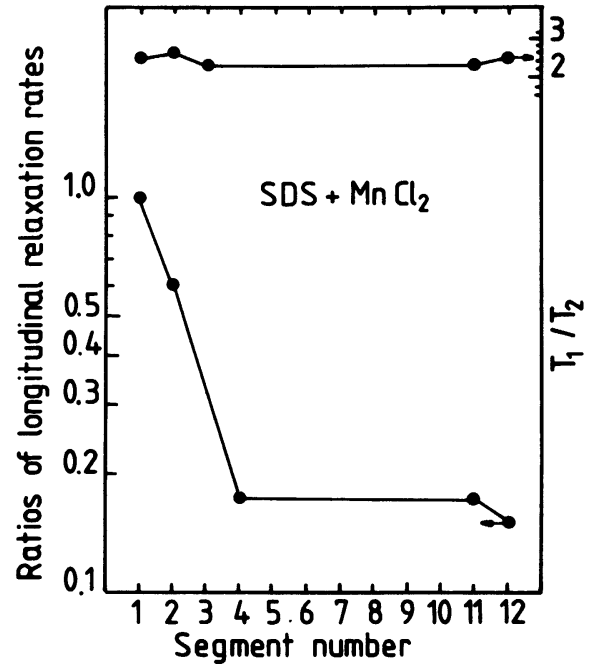

Fig. 9. - Top : values of $T_{1} / T_{2}$ for the relaxation rates produced by $\mathrm{Mn}^{++}$ions on the ${ }^{1} \mathrm{H}$ nuclei in SDS. Bottom : another representation of the ratios shown in figure 8 (compare with Fig. 11). 
to longitudinal relaxation rates has been measured; with $\mathrm{Mn}^{++}$ions this ratio can provide an essential information on the presence of contact interactions and on the magnitude of the correlation times (sections 2.2 and 4.1). Figure 9 shows that this ratio is constant and equal to 2.4 for the whole chain.

3.3 Other ${ }^{13} \mathrm{C}$ relaxation Rates. - The ${ }^{13} \mathrm{C}$ data for SDS with $\mathrm{Mn}^{++}$ions are shown in figure 10 . The values of $1 / T_{2}$ for 0.068 and 0.165 molar SDS indicate that variations of the SDS concentration in this range have no effect on the relaxation rates. The measurements of $1 / T_{1}$ by $T$. Zemb (private commu-

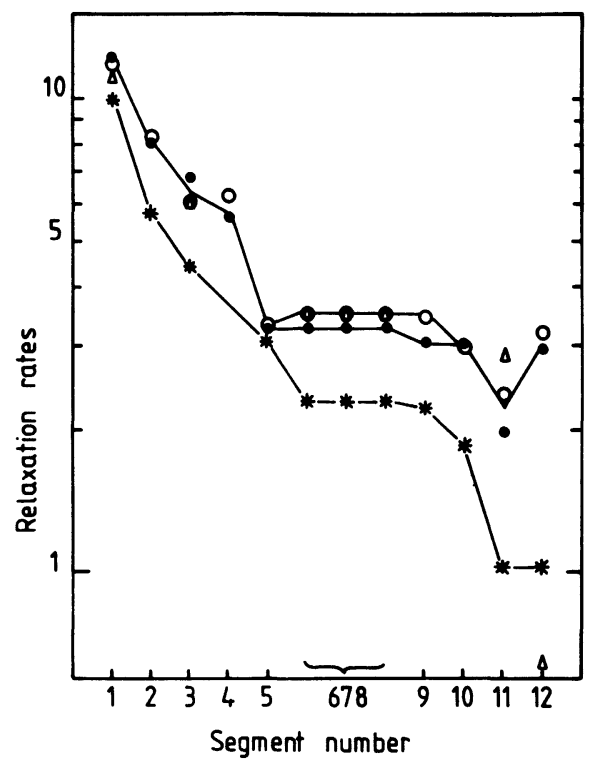

Fig. 10. - Relaxation rates produced by $\mathrm{Mn}^{++}$ions on ${ }^{13} \mathrm{C}$ nuclei in SDS $\left(1 \mathrm{Mn}^{++} / 100\right.$ SDS molecules). Dots : 0.068 molar SDS, values of $1 / T_{2}$ at $69 \mathrm{MHz}$. Circles : 0.165 molar SDS, values of $1 / T_{2}$ at $69 \mathrm{MHz}$. Triangles : 0.165 molar SDS, values of $1 / T_{1}$ at $20 \mathrm{MHz}$. Stars : 0.58 molar SDS, values of $1 / T_{1}$ at $69 \mathrm{MHz}$. The lines are drawn as an aid to the eye. It is argued that a change in micelle shape causes the difference between the curve at high concentration (stars) and the others. nication) on 0.165 molar SDS at a lower frequency $(20 \mathrm{MHz})$ also yield the same values. Consequently the relaxation rates must be frequency independent up to $20 \mathrm{MHz}$; this is to be contrasted with the result obtained at $276 \mathrm{MHz}$ (sect. 3.2) where

$$
1 / T_{1}<1 / T_{2} .
$$

At higher SDS concentration ( 0.58 molar) the value of $1 / T_{1}$, remains unchanged for the first carbon of the chain, but the variation along the chain is different. It is known, however, that for such concentrations the SDS micelles grow from spheres to cylinders $[2,3]$; thus the geometry of the problem is changed, and it is no surprise that different results are obtained.

In figure 11 the ${ }^{13} \mathrm{C}$ data are shown as ratios of relaxation rates, i.e. they have been divided by the

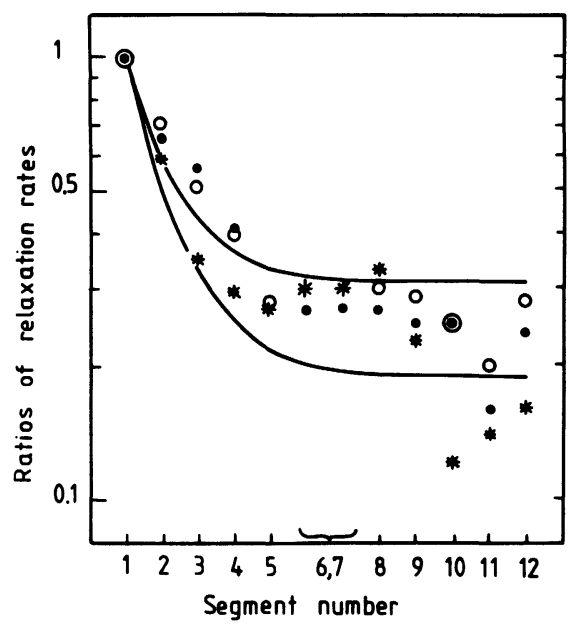

Fig. 11. - Ratios of the contributions of paramagnetic is ns to the relaxation rates of ${ }^{13} \mathrm{C}$ nuclei in SDS micelles. Dots : 0.068 molar SDS with $\mathrm{Mn}^{++}$, values of $1 / T_{2}$. Circles : 0.165 molar SDS with $\mathrm{Mn}^{++}$, values of $1 / T_{2}$. Stars : 0.165 molar SDS with $\mathrm{Co}^{++}$, values of $1 / T_{1}$. The lines drawn correspond to predictions for a dense, random packing of the chains with :- upper line : no water penetration in the hydrocarbon core; - lower line : water penetration in one shell on the core.

Table I. - Assignment of ${ }^{13} \mathrm{C}$ lines in the NMR spectrum of SDS.

$\begin{array}{lc}\text { Carbon } & \\ \text { number } & \text { SDS } 20^{\circ} \mathrm{C} \\ - & - \\ \text { C1 } & 55.51 \\ \text { C2 } & 15.33 \\ \text { C3 } & 11.85 \\ \text { C4 } & 15.82 \\ \text { C5 } & 16.10 \\ \text { C6 } & 16.3 \theta^{-} \\ \text {C7 } & 16.24 \\ \text { C8 } & 15.88 \\ \text { C9 } & 18.32 \\ \text { C10 } & 8.91 \\ \text { C11 } & 0 \\ \text { C12 } & \end{array}$

Shifts (p.p.m. from methyl line $=\mathrm{C} 12$ )

$\begin{array}{cccc}\operatorname{SDS} 40^{\circ} \mathrm{C} & \mathrm{Mg}(\mathrm{DS})_{2} 40^{\circ} \mathrm{C} & \mathrm{Co}(\mathrm{DS})_{2} 40^{\circ} \mathrm{C} & \mathrm{Ni}(\mathrm{DS})_{2} 40^{\circ} \mathrm{C} \\ - & - & 5.44 & 56.15 \\ 55.61 & 55.72 & 15.82 & 15.63 \\ 15.33 & 15.41 & 12.11 & 12.02 \\ 11.80 & 11.89 & 15.77 & 15.79 \\ 15.64 & 15.78 & 16.03 & 16.00 \\ 15.91 & 16.03 & 16.13 & 16.15 \\ 16.07 & 16.21 & 16.07 & 16.08 \\ 16.03 & 16.16 & 15.68 & 15.71 \\ 15.68 & 15.78 & 18.17 & 18.21 \\ 18.19 & 18.28 & 8.81 & 8.83 \\ 8.83 & 8.89 & 0 & 0 \\ 0 & 0 & & \end{array}$


relaxation rate of the first carbon of the chain (closest to the head). These ratios are the same for relaxation rates produced by $\mathrm{Mn}^{++}$and $\mathrm{Co}^{++}$ions; their pattern is also similar to that obtained with protons (Fig. 9), although the exact values are slightly different.

4. Discussion. - 4.1 AdsORPTION OF THE IONS AND NATURE OF THE MAGNETIC INTERACTIONS. All the data for SDS point to a weak adsorption mode, with the ions remaining fully hydrated at the micellar surface and being exchanged at a fast rate between different sites on this surface. Specifically :

- The observed lines will be in the fast exchange regime if the exchange rates of the ions are faster than the relaxation rates and shift frequencies. This is expected to be the case, as the measured relaxation rates are small $\left(<10^{4} \mathrm{~s}^{-1}\right)$ and the shifts are negligible. For the Co/SDS system the lack of temperatulc dependence of the relaxation rates shows that they are indeed in the fast exchange regime. An additional proof of the fast exchange is that the ratios of relaxation rates are the same for micelles with only one $\mathrm{Co}^{++}$and for micelles saturated with $\mathrm{Co}^{++}$ions. For $\mathrm{Mn} / \mathrm{SDS}$, we find the same ratios as with $\mathrm{Co} / \mathrm{SDS}$ : this indicates that this system is in the fast exchange regime as well. An interesting counterexample is found in App. A.

- The next question is that of dipolar vs. contact magnetic interactions. This is easily settled in the case of $\mathrm{Mn}^{++}$ions. Indeed, one finds $T_{1}=T_{2}$ for the ${ }^{13} \mathrm{C}$ of SDS (Figure 10, concentration : $0.165 \mathrm{M}$ ). If contact interactions were present, $T_{2}$ would be much shorter than $T_{1}$ (this has been found for another surfactant, see App. A). With $\mathrm{Co}^{++}$ions, large shifts of the SDS lines would be expected if contact interactions were present; these are not observed (for a counterexample see App. A). Therefore the magnetic interactions are purely dipolar both for Mn/SDS and for $\mathrm{Co} / \mathrm{SDS}$.

- For $\mathrm{Co}^{++}$ions, the relaxation time $\tau_{\mathrm{s}}$ of the electronic spin is quite short (on the order of $10^{-12} \mathrm{~s}$ in a field of $64 \mathrm{kG}$ ). Therefore the fluctuations of the electronic spin control the fluctuations of the dipolar ion-nucleus interaction. The dipolar relaxation rates are then proportional to the $1 / d^{6}$ geometrical factors with a constant scale factor which depends on $\tau_{\mathrm{s}}$. For $\mathrm{Mn}^{++}$ions, the correlation time of the dipolar interaction is known; it can be deduced from the measured values of $T_{1} / T_{2}$ (at $276 \mathrm{MHz}$ the value of $T_{1} / T_{2}$ is 2.4 (Fig. 9), whereas at $69 \mathrm{MHz}$ it is close to 1 (Fig. 10), this yields $\tau_{\mathrm{c}}=0.85 \times 10^{-9} \mathrm{~s}$ ); alternatively one can compare the $T_{1}$ values at these 2 frequencies $\left({ }^{1} \mathrm{H}: 276 \mathrm{MHz} ;{ }^{13} \mathrm{C}: 69 \mathrm{MHz}\right.$-this yields the same value of $\left.\tau_{\mathrm{c}}\right)$. The origin of this correlation time is not as simple as in the case of $\mathrm{Co}^{++}$: the electronic spin relaxation time of $\mathrm{Mn}^{++}$is quite slow $\left(10^{-7} \mathrm{~s}\right.$ in a field of $\left.64 \mathrm{kG}\right)$, and therefore the fluctuations of the dipolar interaction will be controlled by all the relative motions of the ion and nucleus. As discussed in section 2.3.4, the dipolar relaxation rates produced by $\mathrm{Mn}^{++}$ions will not necessarily follow a $1 / d^{6}$ dependence. In practice, one finds that the ratios measured with $\mathrm{Mn}^{++}$ions (Fig. 8) are the same as with $\mathrm{Co}^{++}$ions (Fig. 4), which makes it possible to use either type of ion. So far, however this correspondence between $\mathrm{Mn}^{++}$and $\mathrm{Co}^{++}$has to be taken as an empirical fact, which may not occur for systems other than SDS.

- Knowing the value of $\tau_{\mathrm{c}}$ for $\mathrm{Mn}^{++}$

$$
\left(\tau_{\mathrm{c}}=0.85 \times 10^{-9} \mathrm{~s}\right),
$$

it is possible to calculate the value of $\tau_{\mathrm{s}}$ for $\mathrm{Co}^{++}$ through a comparison of the relaxation rates produced by $\mathrm{Mn}^{++}$and $\mathrm{Co}^{++}$ions. This yields $\tau_{\mathrm{s}}=2.7 \times 10^{-12} \mathrm{~s}$.

- It is then possible to use the Solomon-Bloembergen equations to calculate the average distance $d_{1}$ between the paramagnetic ions and the carbon nuclei $\mathrm{C} 1$ which are directly attached to the head groups of the SDS molecules. There are 2 ways of making this calculation. Either one can assume that the paramagnetic ions have definite adsorption sites on the charged surface, for example directly above one sulfate head group, or in between 3 of them. Then the contributions of all chains which are not directly bound to a paramagnetic ion are neglected. With this drastic approximation the Solomon-Bloembergen equations yield $d_{1}=6 \AA$ for ions bound to one SDS molecule, or $d_{1}=7 \AA$ for ions bound to 3 SDS molecules. Alternatively, one can assume that the density of paramagnetic ions on the charged surface is uniform. Then the equations presented in section 4.2 give the relaxation rate of a nucleus as a function of its depth from the paramagnetic surface; this yields $d_{1}=6 \AA$.

- Thus we can reach a rather precise determination of the distance between the outermost carbon nuclei and the paramagnetic surface; this is because the relaxation rates only depends on $\left(d_{1}\right)^{6}$. On the other hand, the measured relaxation rates are not sensitive to the repartition of the paramagnetic ions on this surface (for example, is it uniform or discrete ?). It is nevertheless interesting to see whether the value of $d_{1}$ is compatible with the picture of fully hydrated ions weakly adsorbed on the micellar surface. In fact, if it is assumed that the ion sits directly above a sulfate head group, then $d_{1}=6$ to $7 \AA$ implies that the ion-sulfur distance is between 4.4 and $5.4 \AA$. This is large enough to allow for water molecules bound both to the head group and to the ion [38]. - Finally the artefacts listed in section 2.3 are not a problem in the present case. First there is no spin diffusion for the ${ }^{1} \mathrm{H}$ decoupled ${ }^{13} \mathrm{C}$ lines. Second, there was the possibility of an overall deformation of the micelle, which would be caused by the ions; this deformation would produce a change in the non magnetic part of the relaxation rates. For SDS concentrations below $0.3 \mathrm{M}$, such deformations are expected to be rather minor. Indeed, the sizes of the micelles 
of CoDS and MnDS are not very different from that of ordinary SDS micelles (section 1.1). In agreement with this, the ratios measured at high divalent ion concentrations are identical with those measured at low divalent ion concentrations (Fig. 4). For SDS concentrations above $0.3 \mathrm{M}$, the micelles become elongated, and their dimensions are quite sensitive to the ionic strength of the solution. This adds a new contribution to the transverse relaxation rates, which depends on the size of the micelle (but not on the position of the nucleus in the chain). Consequently, measurements of $1 / T_{2}$ at high SDS concentrations do not give any information on the configuration of the chains. Third, there was also the possibility of a local deformation of the micellar surface at the adsorption site of a multivalent ion. Again, this is not a problem here because the data obtained on micelles saturated with $\mathrm{Co}^{++}$ions do not differ from those obtained with only one or two $\mathrm{Co}^{++}$ions per micelle.

4.2 Micelle STRUCture. - As explained above, the relaxation rates produced by $\mathrm{Co}^{++}$measure the average of $1 / d^{6}$ over all the respective locations of a nucleus belonging to a SDS molecule and a paramagnetic ion adsorbed on the micelle surface (Fig. 1, sections 2.1 and 4.1). This average has to be expressed as a function of a variable which will describe the configuration of the SDS molecule in the micelle (i.e. with the micelle as a geometrical frame of reference). A good variable for this is the depth of the observed nucleus within the micellar core, or its distance $r$ from the centre of the micelle. If a given nucleus remains located in a thin shell of radius $r$ within the micellar core, and is relaxed by ions adsorbed at a charged surface of radius $R$, its dipolar relaxation rate is :

$$
\frac{1}{T_{1}} \text { or } \frac{1}{T_{2}}=\frac{\kappa}{V} \int_{V} \frac{\delta V}{d^{6}}
$$

where $V=4 \pi r^{2} \delta r$ is the volume of the shell and $\kappa$ a constant given by the Solomon-Bloembergen equations which does not depend upon the geometry if the assumptions of 2.1 hold. Integration yields the simple result :

$$
\frac{1}{T_{1}} \quad \text { or } \quad \frac{1}{T_{2}}=\frac{\kappa\left(R^{2}+r^{2}\right)}{(R+r)^{4}(R-r)^{4}}
$$

The easiest way to use this result is to consider radial vs. non radial chains. For a chain extending radially from the centre of the micelle to its surface, the above equation indicates that the relaxation rates should vary by a factor of 20 between the carbon closest to the head ( $\mathrm{C} 1$, located at $R-r=d_{1}=7 \AA$ ) and the terminal methyl carbon $(\mathrm{C} 12$, at $R-r=22.8 \AA)$. The data shown in figure 2 indicate that SDS chains in spherical micelles are very far from the radial configuration; rather, they suggest that, after a few radial segments, many SDS chains run parallel to the sur- face. Why do they? The basic reason is that the surfactant chains are packed at constant density within the hydrocarbon core of the micelle, and that this prevents most chains from approaching the centre of the micelle. More precisely, a spherical SDS micelle is known to have a hydrocarbon core radius which is equal to the hydrocarbon length of a SDS chain $(15.8 \AA)[4,5]$. This defines the volume of the hydrocarbon core, and therefore the maximum number $N$ of chains which can be packed into this core. The aggregation numbers which are found experimentally are close to $N$, implying that the chains are densely packed within the core. If one defines an « inner core " with a radius equal to one half of the radius of the micellar core, then the volume of this "inner core " is only one eight of the total volume of the micellar core; thus a packing at constant density implies that most chains remain out of this «inner core ».

There are, however, many ways to pack surfactant chains densely in a sphere, and at this point it is appropriate to see whether the data can help to distinguish between various possible packing models. For this purpose it is useful to consider two specific models. Both are "onion shell» models, i.e. they divide the micelle in successive shells whose thickness corresponds to the length per $\mathrm{CH}_{2}$ group in a chain (Fig. 13). These shells are filled with $\mathrm{CH}_{2}$ or $\mathrm{CH}_{3}$ groups according to the following constraints : (i) the density of the packing is constant; (ii) the $\mathrm{CH}_{2}$ groups belong to flexible chains, i.e. the $\mathrm{C} 4$

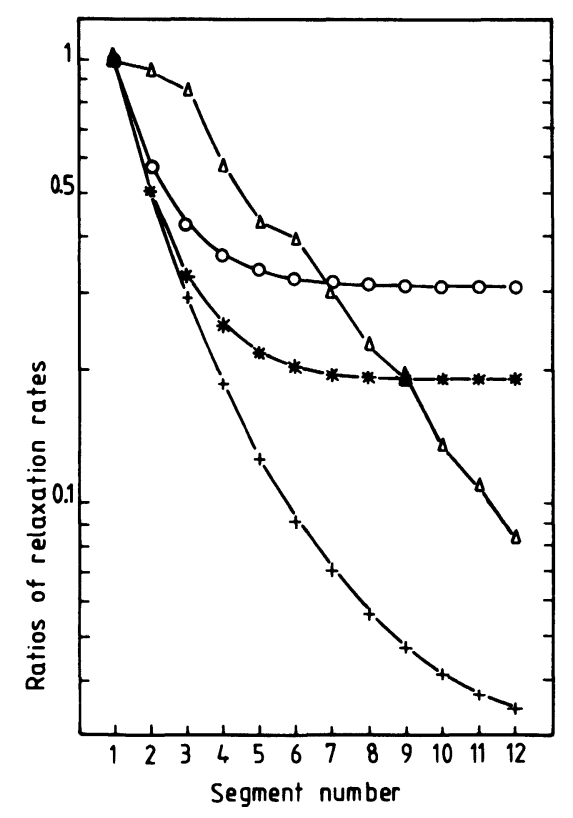

Fig. 12. - Predicted ratios of relaxation rates for various modes of packing of the SDS molecules in a spherical micelle. Crosses : one straight radial chain in a micelle; Circles : dense packing with the $\mathrm{CH}_{3}$ groups scattered throughout the whole hydrocarbon core ; Stars : same as above, but with water penetration in the outermost hydrocarbon shell. Triangles : dense packing with the $\mathrm{CH}_{3}$ groups concentrated near the centre of the core. The radius of the paramagnetic surface is $R=21.8 \AA$, while the radius of the last hydrocarbon shell is $r=15.8 \AA$ (i.e. $d_{1}=6 \AA$ ). 
and $\mathrm{C} 5$ groups of a given chain cannot be located in non adjacent shells; (iii) these chains have one end attached to the outermost shell; (iv) the radius of the outermost shell is equal to the length of the hydrocarbon part of an extended SDS chain.

a

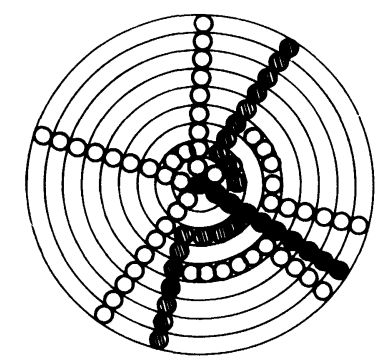

b

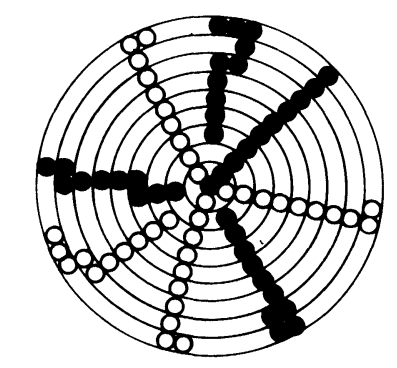

Fig. 13. - Modes of dense packing for surfactant chains in a micelle. a) $\mathrm{CH}_{3}$ groups scattered through the whole hydrocarbon core. b) $\mathrm{CH}_{3}$ groups concentrated near the centre of the core.

The first model is constructed to yield the smallest possible density of terminal methyl groups near the centre of the micelle. This is achieved in the following way. All chains start radially from the surface; at various depths they bend and remain within one shell (Fig. 13a). In this model all the chains who enter the sphere containing the first $n$ shells have $n$ segments in this sphere; the volume of this sphere can accommodate $k n^{2}$ such chains, where $k$ depends on the shape of a $\mathrm{CH}_{2}$ group. From these $\mathrm{kn}^{2}$ chains $k(2 n-1)$ bend and remain in the $n$th shell, and $k(n-1)^{2}$ continue into deeper shells. This model has 2 interesting consequences. First, the mode of packing is the same for every shell, and therefore the same packing can be extended to arbitrary large values of $n$. Second, the terminal methyl groups are distributed over the whole micellar core; there are $k(2 n-1)$ of them in each (nth) shell, up to the surface layer. The same applies to all $\mathrm{CH}_{2}$ groups; for this reason the relaxation rates show a weak dependence upon the position of the observed nucleus in the SDS chain (Fig. 12).

The second model is constructed to yield the highest possible density of terminal methyl groups near the centre of the micelle. For this purpose, the first few shells of the micelle are filled with radial chain segments. In this way, as many methyl groups as possible are used, and in fact the whole supply of methyl groups is exhausted after 8 shells. For the following shells, it is necessary to introduce some kinks or bends of the chains in order to retain a constant density. In this way, the supply of C11 groups is exhausted after the first 9 shells, and so on (Fig. 13b). The resulting ratios of relaxation rates are shown in figure 12 ; near the head of the chain the bends and kinks produce a weak dependence upon the position of the carbon in the chain; at the tail end, the dependence is of course similar to that of a single radial chain in a micelle.

The data shown in figure 2 indicate that the second packing model (Fig. 13b) bears no resemblance to reality. The first model (Fig. 13a) predicts the correct shape of the curve, and the level of the "plateau" is in agreement with the experimental values. Variants of this model can be obtained by assuming that the first shell, or the first 2 shells only contain radial segments (and are therefore not densely packed). This has the effect of increasing the minimal distance between the tails and the paramagnetic surface, and therefore lowering the level of the "plateau ». In this respect, the data presented in figure 2 indicate that the number of these shells in spherical SDS micelles is between 0 and 1. Therefore water/hydrocarbon contact must be limited to the surface of the hydrocarbon core. This conclusion is in agreement with an analysis of thermodynamic data on the hydration of various surfactants [9].

The measured ratios of relaxation rates also provide a definite indication about the "regularity" (or smoothness) of the surface of the micelle. Indeed, an irregular surface would imply that the minimal distance between a given nucleus and the ions would no longer depend on its position along the chain. Therefore the ratios of relaxation rates for the first few carbons would remain close to unity. As this is not the case, it can be concluded that the micelle has a smooth surface.

5. Conclusions. - This work was aimed at devising a method for determining the structure of the water/surfactant interface and the configuration of the surfactant molecules in micelles. It has been shown here that the labelling of micelles with paramagnetic ions can be such a method. In order to obtain unambiguous results, however, it is necessary to choose a system such as $\mathrm{SDS} / \mathrm{Co}^{++}$where :

(i) The paramagnetic ions are weakly adsorbed, remain fully hydrated, and do not deform the micellar surface.

(ii) The magnetic interactions of the nuclei with these ions are purely dipolar.

(iii) The fluctuations of these dipolar interactions are controlled by the fluctuations of the electronic spins of the ions.

In this case the relaxation rates of the nuclei measure a simple geometrical parameter : $\left\langle 1 / d^{6}\right\rangle$ where $d$ is the ion-nucleus distance and the average is taken 
over all positions and configurations of the molecule with respect to the ion. A convenient way to perform this averaging is to divide the micelle into shells; then the relaxation rate of nuclei located in a shell of radius $r$ by ions located in a shell of radius $R$ is proportional to :

$$
\frac{R^{2}+r^{2}}{(R+r)^{4}(R-r)^{4}} .
$$

Thus the ratios of relaxation rates for different nuclei on the chain can be related to the populations of these nuclei in all the shells.

This information is used in the following way : various models for the configurations of the chains in the micelle are constructed, and the corresponding populations of nuclei in the shells are computed. The predicted ratios of relaxation rates of the nuclei are then compared with the experimental ones. Accordingly, the data point to a dense and disordered type of packing, with the chain ends scattered all over the micellar core. Moreover, some of the most commonly depicted modes of packing have to be excluded; in particular, any packing where the tails of the surfactant molecules are radial has to be eliminated.

At this point it is appropriate to recall the original picture drawn by Luzzati [4, 5] from the X-ray work on soap-water mesophases : well organized polar groups forming sharp soap/water interfaces, contrasted with disordered aliphatic chains forming a medium which closely resembles liquid hydrocarbons. The present work brings a quantitative measure of the resemblance between the core of a micelle and a liquid hydrocarbon. First, the data indicate that this core does not contain water. Second, they show that the distribution of chain ends throughout this core is as uniform as the packing constraints will permit (as opposed to a distribution where the chain ends would be located as close to the centre as possible). In other words, the chains minimize their radial extension; if their degree of radial extension is taken as a measure of their order, then the data indicate that the tails are about as disordered as the packing conditions will permit.

More important, however, is the information gained about the geometry of the water/surfactant interface. This interface is composed of 2 regions : a layer which contains the hydrated charges and water, and a layer containing hydrated $\mathrm{CH}_{2}$ groups. The data presented here indicate that the charged surfaces are smooth (in contrast to rough surfaces where some charges lie above and others below the average surface). They also indicate that the water/hydrocarbon contact is limited to the surface of the hydrocarbon core (i.e. the thickness of the layer containing the hydrated $\mathrm{CH}_{2}$ groups is comparable to the length per $\mathrm{CH}_{2}$ group in a chain). Other interfaces of practical importance could be studied through this technique; it would be particularly appropriate for interfaces swollen with solutes (polymers dyes, alcohols) where the location of the solutes within the interface is not known. The main limitation of this technique will again be the need to label the interface with divalent paramagnetic ions; on the other hand, if this labelling can be used, it will provide a measurement of distances within the interface with a resolution not attainable by other techniques.

Acknowledgments. - It is a pleasure to thank M. Guéron, J. L. Leroy and P. Plateau for the use of their NMR spectrometer and for many discussions. I also thank T. Zemb for permission to use the results of his low frequency $T_{1}$ measurements on the ${ }^{13} \mathrm{C}$ lines of SDS (Fig. 10), as well as for valuable suggestions concerning the interpretation of the data.

Appendix A : Strong binding of $\mathrm{Co}^{++}$ions to sodium octanoate. - In the course of this study, one type of surfactant molecule (carboxylates) was" found which binds strongly to the paramagnetic ions (as opposed to the sulfates, whose binding is weak). The experiments are as follows : $\mathrm{CoCl}_{2}$ is added to a 0.023 molar solution of $\mathrm{K}$ octanoate (this is well below the c.m.c. of $\mathrm{K}$ octanoate, which is $0.34 \mathrm{M}$ ); the NMR lines of the ${ }^{1} \mathrm{H}$ nuclei of the octanoate are observed. Upon addition of $\mathrm{Co}^{++}$ions, the line of the first $\mathrm{CH}_{2}$ group (closest to the carboxyl) undergoes a large shift and a very large broadening (Figs. 14 and 15); the longitudinal relaxation rate created by the ions is more modest, yielding a high value of $T_{1} / T_{2}(\sim 300)$. All these effects are proportional to the concentration of $\mathrm{Co}^{++}$ions, and show no sign of saturation, even for compositions up to $80 \mathrm{Co}^{++} / 100$ octanoate (Fig. 14). Accordingly, only a small fraction of the $\mathrm{Co}^{++}$ions is bound to the

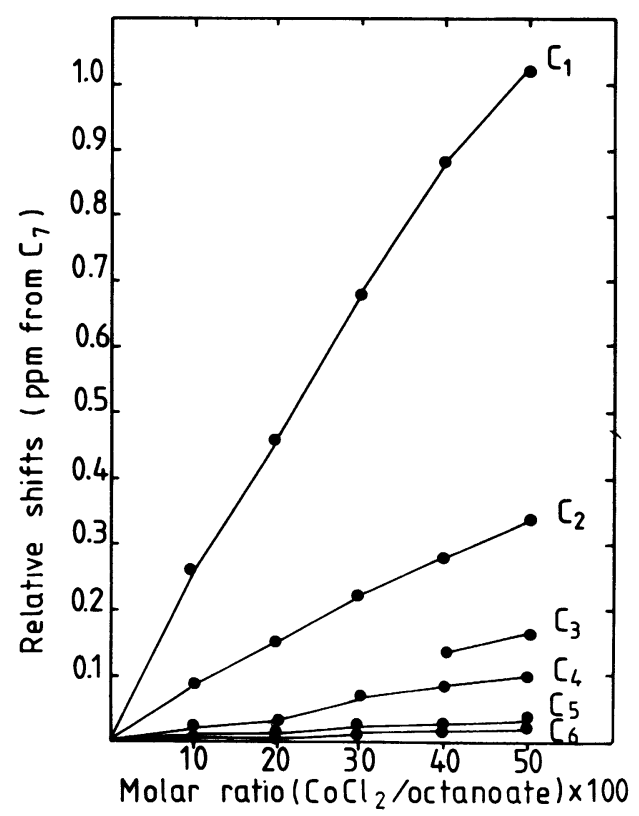

Fig. 14. - Effect of $\mathrm{Co}^{++}$ions on the chemical shift difference between each ${ }^{1} \mathrm{H}$ nucleus of potassium octanoate and the methyl ${ }^{1} \mathrm{H}$. 
octanoate, and most ion pairs are dissociated; this would not be the case if micelles or premicellar aggregates were formed. The other $\mathrm{CH}_{2}$ of the octanoate chain are also shifted with respect to each other, and these shifts appear to decrease exponentially along the chain. Their broadenings are much less important than for $\mathrm{C} 1$ (Fig. 15). Finally the longitudinal relaxation rates show a regular decrease along the chain (Fig. 15).

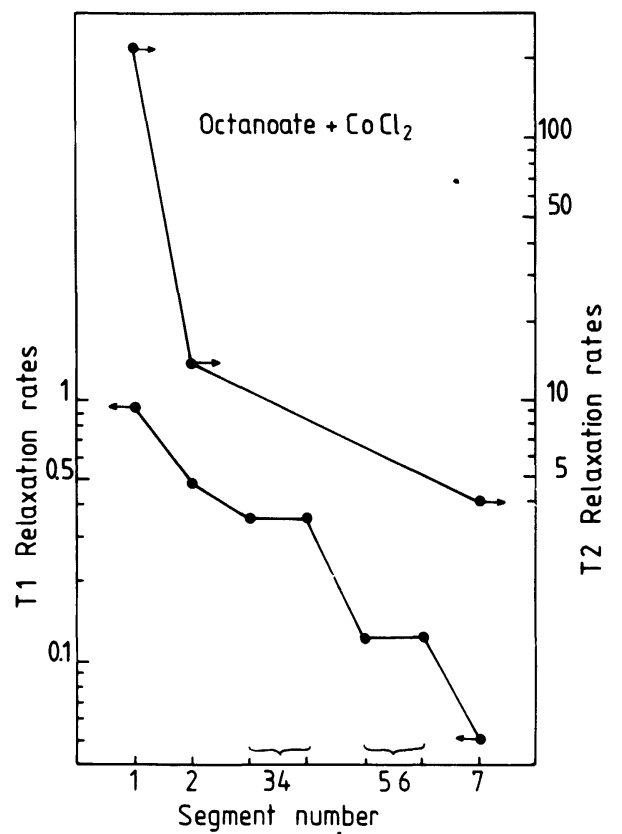

Fig. 15. - Top : contribution of $\mathrm{Co}^{++}$ions to $1 / T_{2}$ for ${ }^{1} \mathrm{H}$ nuclei in potassium octanoate $\left(20 \mathrm{Co}^{++} / 100\right.$ octanoate). Bottom : contribution of $\mathrm{Co}^{++}$to $1 / T_{1}$.

As before, the first question to settle is that of the exchange regime for these ${ }^{1} \mathrm{H}$ lines (fast, intermediate, or slow ?). For the $\mathrm{CH}_{2}$ closest to the carboxyl, a comparison of the shift and broadening shows that the linewidth $\left(1 / T_{2}\right)$ is in the intermediate exchange regime, whereas the longitudinal relaxation is in the fast exchange regime. For the other $\mathrm{CH}_{2}$ groups, the exchange is fast both for $1 / T_{1}$ and for $1 / T_{2}$. Next, it is necessary to identify the mechanism of the magnetic interactions. The ratios of the shifts show that they cannot have a dipolar origin; therefore they must be produced by a contact interaction. Consequently the linewidth of the first $\mathrm{CH}_{2}$, which is controlled by the shift, also has a contact origin. On the other hand, the $T_{1}$ ratio for the first and second $\mathrm{CH}_{2}$ are consistent with the geometry of the octanoate $/ \mathrm{Co}^{++}$complex, assuming a distance of $2.8 \AA$ between the ion and the carbonyl; this, together with the small values of $1 / T_{1}$, indicates that a dipolar mechanism controls the $T_{1}$ values.

Thus there are 2 indications that the $\mathrm{Co}^{++}$ions lose some of their hydration water upon binding to the octanoate. First, a contact interaction is observed on the methylene groups closest to the carboxyl. Second, the ion-carboxyl distance obtained from the
$T_{1}$ relaxation rates $(2.8 \AA)$ corresponds to a direct binding. That $\mathrm{Co}^{++}$ions bind directly to carboxylates but not to sulfates is consistent with their ability to precipitate these surfactants : $\mathrm{K}$ octanoate above its c.m.c. is precipitated by $\mathrm{CoCl}_{2}$, whereas SDS is not. A similar conclusion has been reached by Karenzi, Meurer, Spegt and Weill in a study of the binding of paramagnetic ions to polyelectrolytes [36] : they find that the ions bind to the $\mathrm{SO}_{3}^{-}$groups of polystyrenesulfonate without losing any of their hydration water, whereas they do become partially dehydrated upon binding to the $\mathrm{CO}_{2}^{-}$groups of polyacrylate. That study also suggests an explanation for the difference in binding behaviour to various anions : the binding could be controlled by the strength of the local electric field, which depends upon the distance between the ions and the hydrocarbon/water interface; this distance is much larger for sulfates than for carboxylates. Indeed, it has long been known that surfactant molecules where the charged groups are widely separated from the hydrocarbon show a remarkable resistance to precipitation by divalent ions [37].

Appendix B : Measurements on alkyl sulfate solutions below their critical micelle concentrations. A micellar SDS solution contains, in equilibrium with SDS micelles, a small number of unassociated $\mathrm{DS}^{-}$ions. As these $\mathrm{DS}^{-}$ions bear a single charge, it is expected that they will bind very few divalent counterions such as $\mathrm{Co}^{++}$or $\mathrm{Mn}^{++}-$i.e. the ion pairs $\mathrm{Co}^{++} / \mathrm{DS}^{-}$should be largely dissociated, as opposed to the micellar species $\left(\mathrm{Co}^{++}\right)_{x}\left(\mathrm{DS}^{-}\right)_{y}$ for which the counterions are associated to a large extent. Nevertheless, it is necessary to ensure that these unassociated $\mathrm{DS}^{-}$ions do not contribute to the paramagnetic relaxation rates. This can be done by measuring the effect of $\mathrm{Mn}^{++}$ions on the relaxation rates of SDS solutions at concentrations well below the c.m.c.; in this case all the $\mathrm{DS}^{-}$ions are

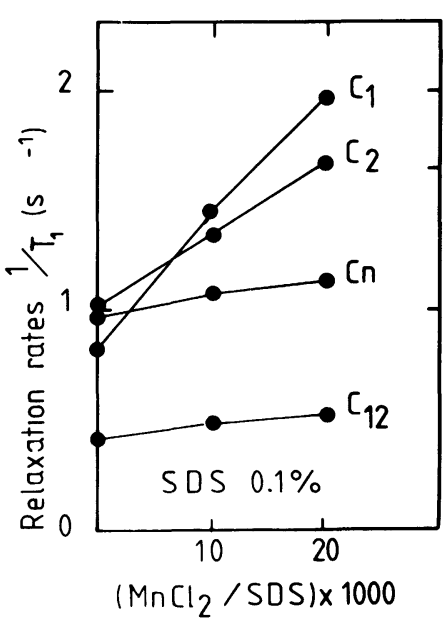

Fig 16. - Effect of $\mathrm{Mn}^{++}$ions on the values of $1 / T_{1}$ for a SDS solution below the c.m.c. These relaxation rates are 2 orders of magnitude smaller than those measured above the c.m.c. (Fig. 7). 
unassociated. The ${ }^{1} \mathrm{H}$ relaxation rates for a 0.0034 molar SDS solution are shown in figure 16; a comparison with figure 7 indicates that they are much smaller than the corresponding rates for a micellar solution of SDS. Thus it is quite safe to assume that the contribution of unassociated $\mathrm{SDS}^{-}$ions to the relaxation rates in a micellar SDS solution is negligible.

In addition, the relaxation rates of unassociated alkylsulfate ions provide some information about their average configuration in water. Just as in the case of micellar solutions, these relaxation rates are produced by dipolar interactions. There are, however, two major differences. First, the tumbling rate of small isolated molecules such as sodium butylsulfate (SBS) is faster $\left(\sim 2 \times 10^{-11} \mathrm{~s}\right)$ than the time for internal motions of the molecule $\left(\sim 10^{-10} \mathrm{~s}\right)$. Therefore in the case of the Mn/SBS system, the dominant correlation time will be the rotation of the ion pair (this is not true for Mn/SDS below its c.m.c., as tumbling and internal motions will both be on the order of $\left.10^{-10} \mathrm{~s}\right)$. Second, the paramagnetic relaxation can no longer be produced by ions adsorbed on neighbouring molecules, as in the case of micelles. Consequently, the relaxation rates of short isolated molecules such as SBS measure the average of $1 / d^{6}$,

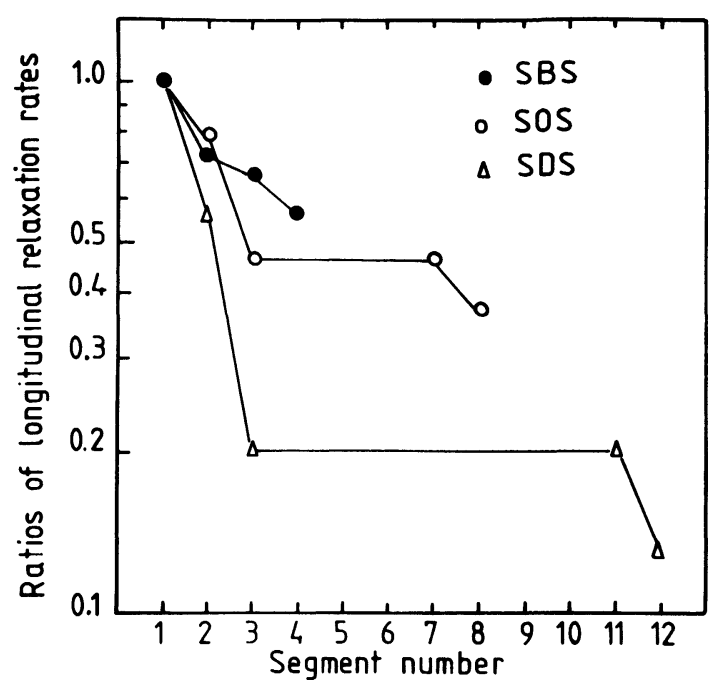

Fig. 17. - Ratios of the contributions of $\mathrm{Mn}^{++}$to $1 / T_{1}$ for ${ }^{1} \mathrm{H}$ nuclei in alkylsulfates below the c.m.c.

where $d$ is the distance between the observed nucleus and the ion adsorbed on the polar group of the molecule. The measured ratios of relaxation rates (Fig. 17) indicate that isolated alkyl chains in water tend to adopt a range of folded configurations and probably minimize the hydrocarbon/water contact in this way.

References

[1] Hartley, G. S., Aqueous solutions of paraffin chain salts (Herrmann, Paris) 1936

[2] Mysels, K. J., J. Colloid. Sci. 10 (1955) 507

[3] Mysels, K. J., Princen, L. H., J. Phys. Chem. 63 (1959) 1696.

[4] Reiss Husson, F., Luzzati, V., J. Colloid Interface Sci. 21 (1966) 534

[5] Reiss Husson, F., Luzzati, V., J. Phys. Chem. 68 (1964) 3504.

[6] Luzzati, V., Mustacchi, H., Skoulios, A., Husson, F., Acta Crystallogr. 13 (1960) 660 and 668.

[7] Cabos, C., Delors, P., J. Physique 39 (1978) 432.

[8] Rosenholm, J. B., Drakenberg, T., Lindman, B., J. Colloid Interface Sci. 63 (1978) 538.

[9] Wennerström, H., Lindman, B., J. Phys. Chem. 83 (1979) 2931.

[10] Podo, F., Ray, A., Nemethy, G., J. Am. Chem. Soc. 95 (1973) 6164.

[11] Rosenholm, J. B., Stenius, P., Danielsson, I., J. Colloid Interface Sci. 57 (1976) 551.

[12] Kalyanasundaram, K., Thomas, J. K., J. Phys. Chem. 80 (1976) 1462.

[13] Henriksson, U., Odberg, L., Eriksson, J. C., Westman, L., J. Phys. Chem. 81 (1977) 76.

[14] Abragam, A., The principles of nuclear magnetism (Oxford, U.P.) 1961.

[15] DWECK, R. A., NMR in biochemistry (Clarendon, Oxford) 1973.

[16] Cabane, B., in Magnetic resonance in colloid and interface science, Nato Adv. Study Inst. Series, vol. 61 (D. Reidel, Dordrecht) 1980.

[17] Fox, K. K., Robi, I. D., Smith, R., J. Chem. Soc. Faraday I. 68 (1972) 445.

[18] Hauser, H., J. Colloid Interface Sci. 55 (1976) 85.

[19] Van Bockstaele, M., Gelan, J., Martens, H., Put, J., de Schryver, F. C., Deredon, J. C., Chem. Phys. Lett. 70 (1980) 605.

[20] Satake, I., Iwamatsu, I., Hosokawa, S., Matuura, R., Bull. Chem. Soc. Japan 36 (1963) 204.

[21] Moroi, Y., Motomura, K., Matuura, R., J. Colloid Interface Sci. 46 (1974) 111.
[22] Rohde, A., Sackmann, E., J. Colloid Interface Sci. 70 (1979) 494.

[23] Mazer, N. A., Benedek, G. B., Carey, M. C., J. Phys. Chem. 80 (1976) 1075.

[24] Cabane, B., J. Phys. Chem. 81 (1977) 1639.

[25] Roberts, R. T., Chachaty, C., Chem. Phys. Lett. 22 (1973) 348.

[26] Germain, C., Cabane, B., to be published.

[27] For example the correlation time for the motion of $\mathrm{VO}^{++}$ ions adsorbed on SDS micelles is $7.5 \times 10^{-11} \mathrm{~s}$, as measured by :

Stilbs, P., Jermer, J., Lindman, B., J. Colloid Interface Sci. 60 (1977) 232.

[28] Solomon, I., Phys. Rev. 99 (1955) 559.

[29] Solomon, I., Bloembergen, N., J. Chem. Phys. 25 (1956) 261

[30] Swift, T. J., Connik, R. E., J. Chem. Phys. 37 (1962) 308.

[31] Luz, Z., Мeiвoом, S., J. Chem. Phys. 40 (1964) 2686.

[32] Shulman, R. G., Sternlicht, H., Wyluda, B. J., J. Chem. Phys. 43 (1965) 3116.

[33] Sternlicht, H., Shulman, R. G., Anderson, E. W., J. Chem. Phys.

[34] Espersen, W., Martin, R., J. Phys. Chem. 80 (1976) 161

[35] ABdolall, K., Ph. D. Thesis, University of British Columbia (1978).

[36] Karenzi, P. C., Meurer, B., Spegt, P., Weill, G., Biophys. Chem. 9 (1979) 181.

[37] Mato, M., Shinoda, K., J. Phys. Chem. 77 (1973) 378.

[38] That $\mathrm{Mn}^{++}$ions remain fully hydrated is confirmed by the results of EPR experiments [39]. Indeed, the EPR line of $\mathrm{Mn}^{++}$ions is only moderately broadened by the addition of SDS, whereas a dehydration of these ions would break the symmetry of their environment and create much more severe effects [36]. On the other hand, other ions may behave differently. For example, $\mathrm{Ni}^{++}$ions seem to produce measurable contact interactions on the first carbon of the SDS molecule $; \mathrm{Ca}^{++}$ions are much more efficient than other divalent ions in precipitating SDS, which may be an indication of a partial dehydration of the ion.

[39] OAKES, J., J.C.S. Faraday II 69 (1973) 1321. 